\title{
Recording, Monitoring and Interrelating Changes of Invivo Bio-cells from Video (Biosignatures)
}

Nikolaos Bourbakis

ITRI, WSU, College of Engineering, Dayton, OH 45435

AIIS Inc., nbourbakis@woh.rr.com

\begin{abstract}
This paper presents a synergistic methodology for automatically recording, monitoring and interrelating changes occurred in invivo bio-cells without any user's assistance. The methodology presented here combines several techniques, such as projection functions, registration, segmentation with region synthesis, local-global graphs and stochastic Petrinets. Each of these techniques produces complementary results and the synergistic combination of them generates a methodology that produces the bio-signatures of bio-cells in sequences of images. Illustrative results are also provided.
\end{abstract}

\section{Introduction}

Detecting and recording changes in different images captured from the same scene at different time and orientation is a subject of important interest in the area of medical imaging. Several methodologies have been proposed with good results [110]. Most of these methods use quantitative difference measures and a wide list of quantitative image difference measures can be found in [1]. The measures come mainly from signal processing area and do not take into account an image interpretation. Let we name at least some of them. Given two images $1, J$, both of size $M \times N$, the following measures were defined [1].

Maximum Difference $\quad M D=\max \left[I_{i j}-J_{i j} \mid\right.$;

Normalized Average Difference $N A D=\left(1 / \sum\left|I_{i j}\right|\right)\left[\sum\left|I_{i j}-J_{i j}\right|\right]$;

Average Difference $\quad A D=(1 / M N) \sum\left|I_{i j}-J_{i j}\right|$;

Mean Square Error $\quad M S E=(1 / M N) \sum\left(I_{i j}-J_{i j}\right)^{2}$;

Normalized Mean Square Error NMSE $=\left[1 / \sum\left(I_{i j}\right)^{2}\right]\left[\sum\left(I_{i j}-J_{i j}\right)^{2}\right]$;

Peak Signal to Noise Ratio $P S N R=\operatorname{lolog}_{10}\left(I^{2} \max / M S E\right)$;

where $I_{\max }$ is a maximum possible pixel value.

There also methods for detecting changes in images and they are related to the human visual system (HVS), where its sensitivity depends on viewing conditions, namely on image illumination, background illumination, viewing distance, color fidelity. Those conditions must be usually fixed while evaluating an image distance

Please use the following format when citing this chapter:

Bourbakis, Nikolaos, 2006, in IFIP International Federation for Information Processing, Volume 204, Artificial Intelligence Applications and Innovations, eds. Maglogiannis, I., Karpouzis, K., Bramer,

M., (Boston: Springer), pp. 467-475 
measure. An interesting approach is proposed in [2]. They choose the viewing distance adaptively for every image so that the image of an object appears at the peak of the HVS sensitivity curve, or about 8 cycles per degree. The quality measure is then based on the background illumination level sensitivity and the spatial frequency nonlinear models. Moreover, a simple and in some degree valuable model to insure that image changes produced in their process of embedding a piece of information into an image is visually transparent is used in [3]. The S-CIELAB standard is followed to transform each image into an opponent-color representation. Then apply low-pass filtering to each of the 3 bands and the result transform into CIE XYZtristimulus coordinates. Finally, the CIELAB formula for color differences $\triangle \mathrm{E}$ is evaluated (its definition can be found in [4]. A value of $\triangle E$ less than 1 implies that the differences are not detectable by humans. S-CIELAB requires some calibration parameters like viewing distance (angle), white point, and the mapping between the digital representation of the color images and the spectral reflectance of the displayed images.

In addition, a method based on local global (L-G) graphs for efficiently representing the cell's structural features (shape, size, color, texture, regions) and to some degree the changes occurred in different consecutive images has been presented in [10]. Thus, the new contribution in this paper is the correlation and interrelation of the detected changes for automatically generating bio-signatures or contributing to an interpretation of the events or actions that took place in the sequence of images [9].

\section{The Bio-signature based Method}

This method is based on the synergistic combination of several techniques, such as pixels projections [7], registration [8], region-grown segmentation with region synthesis [6], local-global graphs [5], Stochastic Petri nets (SPN) [9]. In particular, the pixels projection functions offer an efficient capability for detecting changes in 2D images captured from the same altitude and the same direction. The image registration is offering the capability for matching same regions in images taken from different scale, translation and rotation. The image segmentation contributes the clustering of different regions with certain characteristics, such as same of similar color, texture and the synthesis of regions composes regions with similar features into larger regions that serve the goals of the criteria selected by the user or a particular application. The L-G graphs provide an accurate representation of the regions structural features. Finally the SPN model offers to the synergy operational features, such as timing, synchronization, parallelism, concurrence of events that occurred in consecutive images captured by a video camera [9].

\subsection{Pixels Projection Functions}

The pixels projection functions are based on the Radon transformation:

$$
\begin{aligned}
& T(\rho, \theta)=R_{\theta}[f(x, y)]= \\
& \iint f(x, y) \cdot \delta(\rho-x \cos \theta, \rho-y \sin \theta) d x d y .
\end{aligned}
$$

$\rho, \theta$ are the spatial variables in the radial coordinates, $\rho$ is the radium and $\theta$ the angle, 
and $\delta$ is the Kronecer's delta function defined as: $\delta_{\mathrm{ij}}=1$ if $\mathrm{i}=\mathrm{j}$, and $\delta_{\mathrm{ij}}=0$ if $\mathrm{i} \neq \mathrm{j}$. Then the Radon transformation is expressed as

$$
T_{0^{\circ}}(y)=\sum_{x}^{N r} I(x, y) \quad T_{0^{\circ}}(y)=\sum_{x}^{N r} I(x, y)
$$

The result of this process is the decomposition of the initial 2-D signal into two 1-D signal.

\subsection{Registration}

Image registration is the process of geometrically or topologically aligning two images of the same scene that have been taken under varying conditions. It is usually employed as the first stage in medical imaging. For the case of change detection, the objective is to find the differences between two images of the same scene that have been taken from variable viewpoints, at different times, using different cameras. Figure 1 illustrates the image registration process in two bio-images.

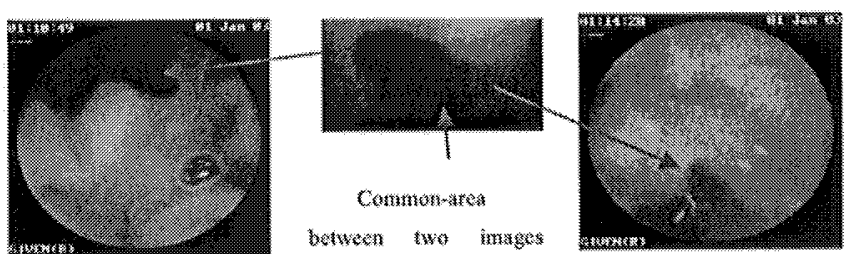

Figure 1: It shows two WCE images taken from different directions and different time and the $91 \%$ mapping of a possible common areas after registration.

\subsection{Region Growing Image Segmentation}

Here, we use the Fuzzy-like Reasoning Segmentation (FRS) method with Region Synthesis that adds light model as one of the segmentation factors. Its result is more accurate in terms of perception and more suitable for later reconstructing work. The FRS method has three stages (smoothing, edge detection and segmentation). The initial smoothing operation is intended to remove noise. The smoother and edge detector algorithms are also included in this processing step [6]. The segmentation algorithm uses edge information and the smoothed image to find segments present within the image. Figure 2 shows a segmented image and the synthesis of regions based on predefined recognition criteria.
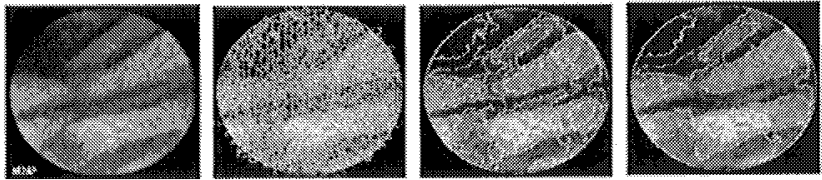

Fig. 2: It illustrates the results from an image segmentation and the synthesis of regions with similar features generating the desirable region-object

\subsection{L-G Graphs}

The Local-Global $(L-G)$ graph method combines local-regional information with global topological representation of information distributed in an image [7]. The graph is a more accurate representation of an object. Thus, we avoid using a non- 
linear graph matching function. By combining the FRS method and the $L-G$ graph method, we can improve object recognition accuracy without increasing computation complexity. Thus, here the L-G graph is capable of describing with adjustable accuracy and robustness the features contained in an image. The main components of the L-G graph are: (i) the local graph that represents the information related with color, texture, shape, size, (ii) the skeleton graph that provides in formation about the internal shape of each segmented region, and the global graph that represents the relationships among the segmented regions for the entire image. Thus, the basic idea behind this graph based method is the local and global geometric representation of the image features and their relationships. The selection of the graph structure for representing the information extracted from an image is important for two reasons: 1) it is a generic and very flexible information representation scheme; 2) it is very robust and computationally not very expensive.

\subsection{Stochastic Petri-net Graphs}

The graph models mentioned above have the capability of holding structural information for objects. Thus, the missing element is the functional behavior of a object. The functional behavior of an object is described by the states that the object could be transferred after an appropriate triggering. A successful and power model capable of describing (or modeling) the functional behavior of a system is the Stochastic Petri-net (SPN) model. Thus, in order to maintain the structural features of the graph model and the functional features of the SPN model, a mapping is presented here, where the SPN model is transformed into a SPN graph model as follows [9]:

\section{$m:$ G $\rightarrow$ SPNG}

where, $\{\mathbf{N i}\} \rightarrow\{\mathbf{P i}$, graph-nodes correspond into SPN places, and \{aij\} $\{$ tij\}, relationships corresponds into SPN transitions.

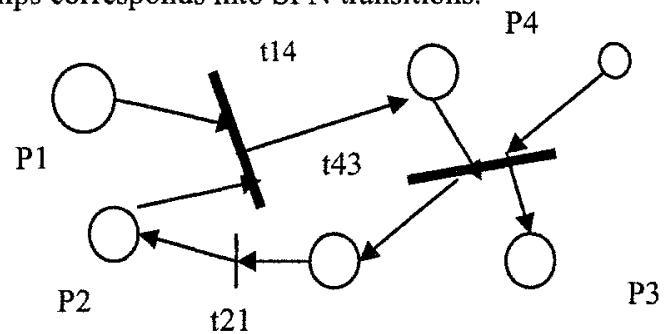

The SPNG above illustrates an object that has four different states (Places Pi, $i=1,2,3,4)$. Each place $P i$ has its own structural features transferred from the corresponding graph node $\mathrm{Ni}$. The transitions 114 and $\mathrm{t} 43$ represent relationships among the same parts of an object and a stochastic distribution of time required to fire that transition. The transition $\mathbf{t} 21$ requires no time to fire.

\subsection{Illustrative Examples}

In this section we present illustrative examples of bio-images taken from a sequence of images (especially two at a time) for showing the results of the changes detection methods. The examples 1 and 2 show results for detecting changes in two images using the Radon transformation with horizontal, vertical and diagonal projections. 
The changes were detected by combining the horizontal, vertical and diagonal projections. This methods alone is good for images where rotation and scaling don't occurred. The example-3 presents the structural and the functional parts of the biosignatures extracted from the changes occurred in invivo cells from a sequence of images.

\section{Example-1: Two images taken from the same region with differences}

Original Images

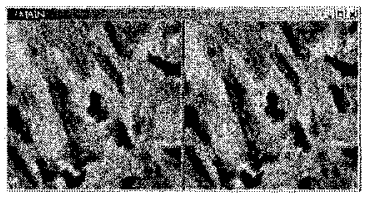

Projections (horizontal, vertical)
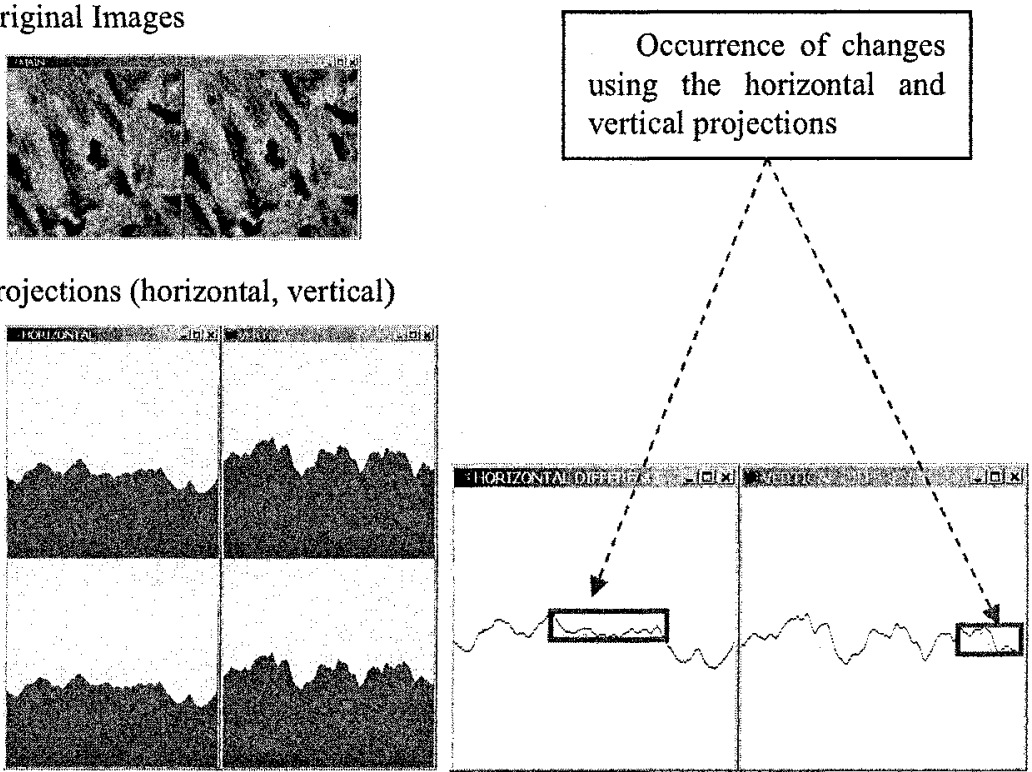

\section{Projections (diagonal)}

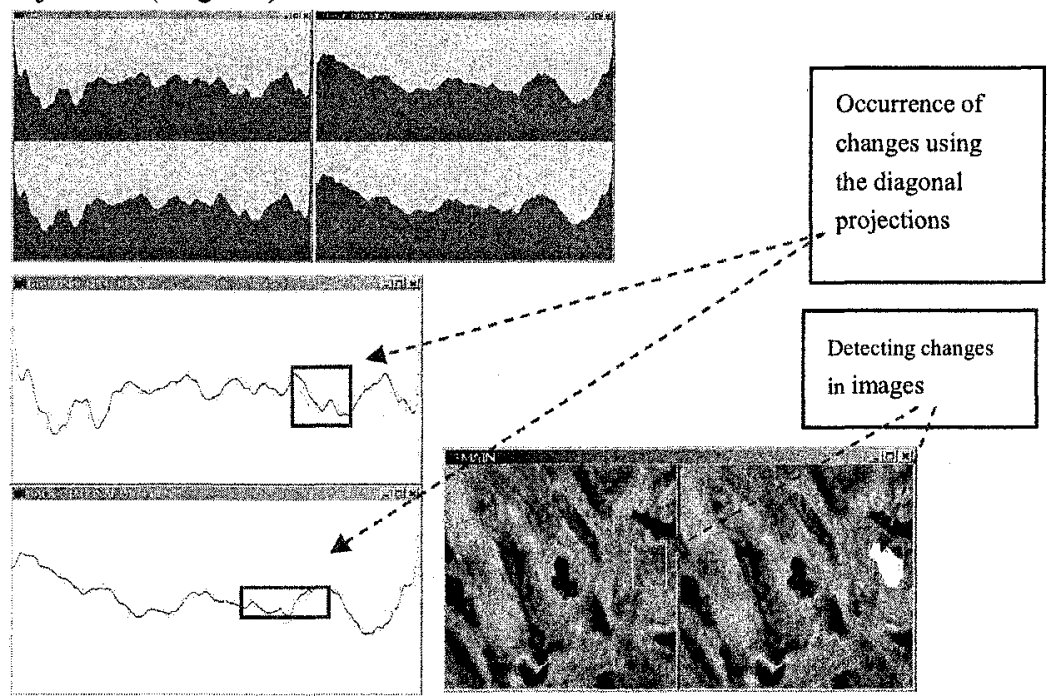


Example-2: Changes detected in images taken from the same region but in different times
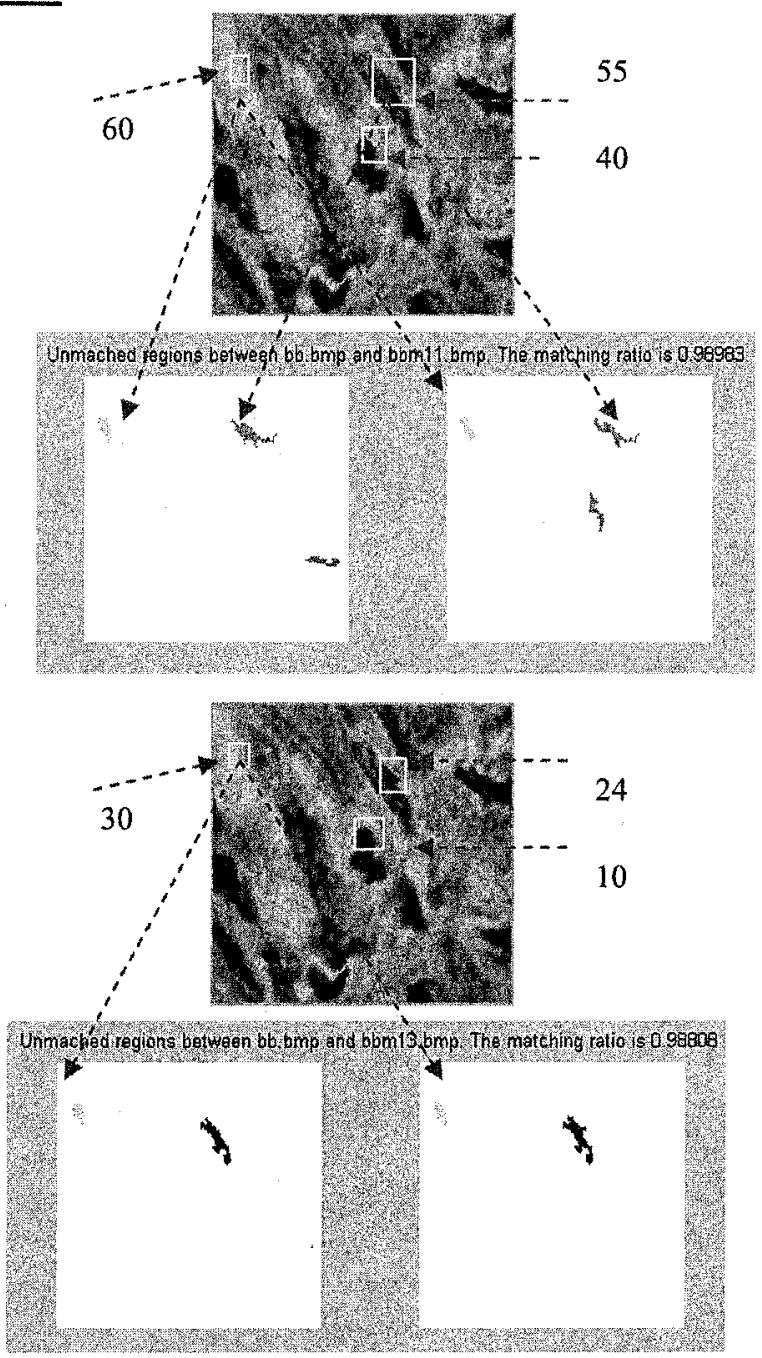

\section{Example-3: Biosignatures.}

Here we present the Bio-Signatures structural part using Local Global (L-G) graphs and SPN graphs. The L-G graphs extract the structural features (size, shape, color, texture, and centroid) of each cell's region and the SPN graphs associate these structural features with their changes in time (states), by creating the functional parts of the biosignatures. The illustrative example below shows a region and the extracted featues. 


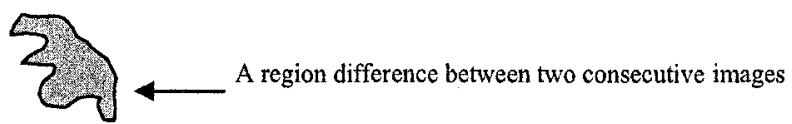

Size: 3,251 pixels

Shape:

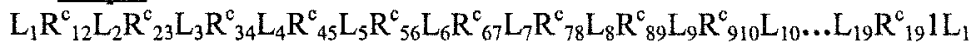

Graph: $\mathrm{N}_{1} \mathrm{a}_{12}^{\mathrm{c}} \mathrm{N}_{2} \mathrm{a}^{\mathrm{c}}{ }_{23} \mathrm{~N}_{3} \mathrm{a}^{\mathrm{c}}{ }_{34} \mathrm{~N}_{4} \ldots \mathrm{N}_{19} \mathrm{a}^{\mathrm{c}}{ }_{191} \mathrm{~N}_{1} @ \mathrm{~N}_{2} \mathrm{a}^{\mathrm{p}}{ }_{215} \mathrm{~N}_{15} \mathrm{a}^{\mathrm{p}}{ }_{1518} \mathrm{~N}_{18} @ \ldots$

(a) $\mathrm{N}_{2} \mathrm{a}^{\mathrm{v}}{ }_{28} \mathrm{~N}_{8}$

$\mathrm{RGB}$ values
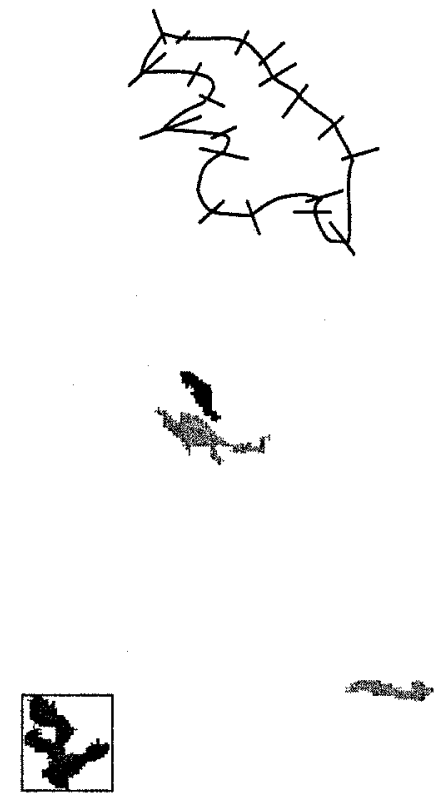

1.
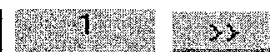

Show hro

The figure above shows the cell's regions detected with differences from their previous status by using software tool's. One region was then selected and frame for extracting its features. Also the features extracted from two region are shown below.

Region (1) into

RGB wite: $R=41 \quad G=41 \quad B=41$

Region size is 6 ib

Region centrid (193.6641 99.9891)

WWW Fegen $(1 \omega)$ frito

RGE value $R=67 \quad G=67 \quad B=67$

Fegion size is 595

Fegin tentrid (120.0879 123.9925)

W
Rejom (2) ino

RTS vilue: $R=132 \quad G=132 \quad B=132$

Pegion size is 314

Rezion centroid (62.293 162.2707)

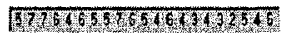

Fegion (2-) info

RGB yalue $R=139 \quad G=139 \quad B=139$

Fegion size is 278

Region centrodd (51.4167 152.5507)

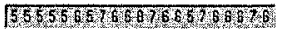


The figure below shows the graphical representation (in time) of the changes occurred in regions of a particular cell.

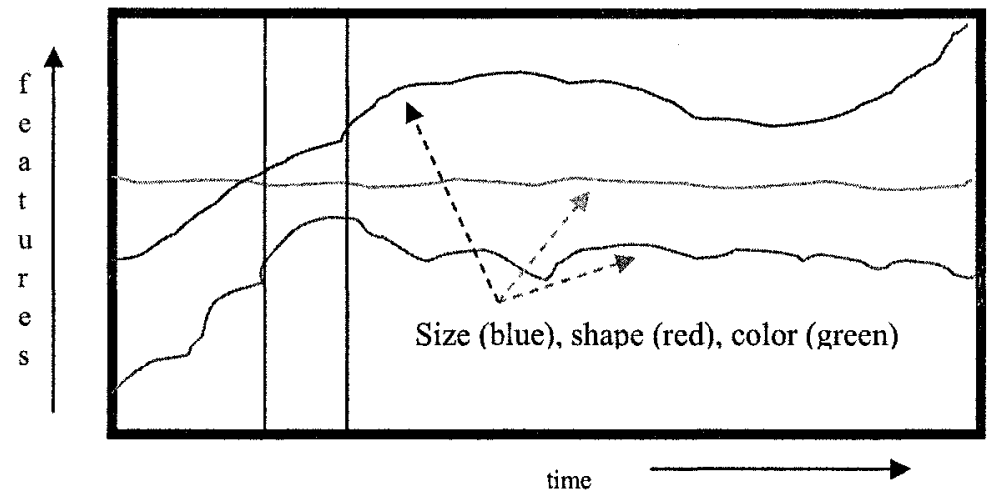

The last figure presents the interrelations among the changes that took place in the same cell by using the SPN graphs.

SPN graphs (bio-signatures, functional part)

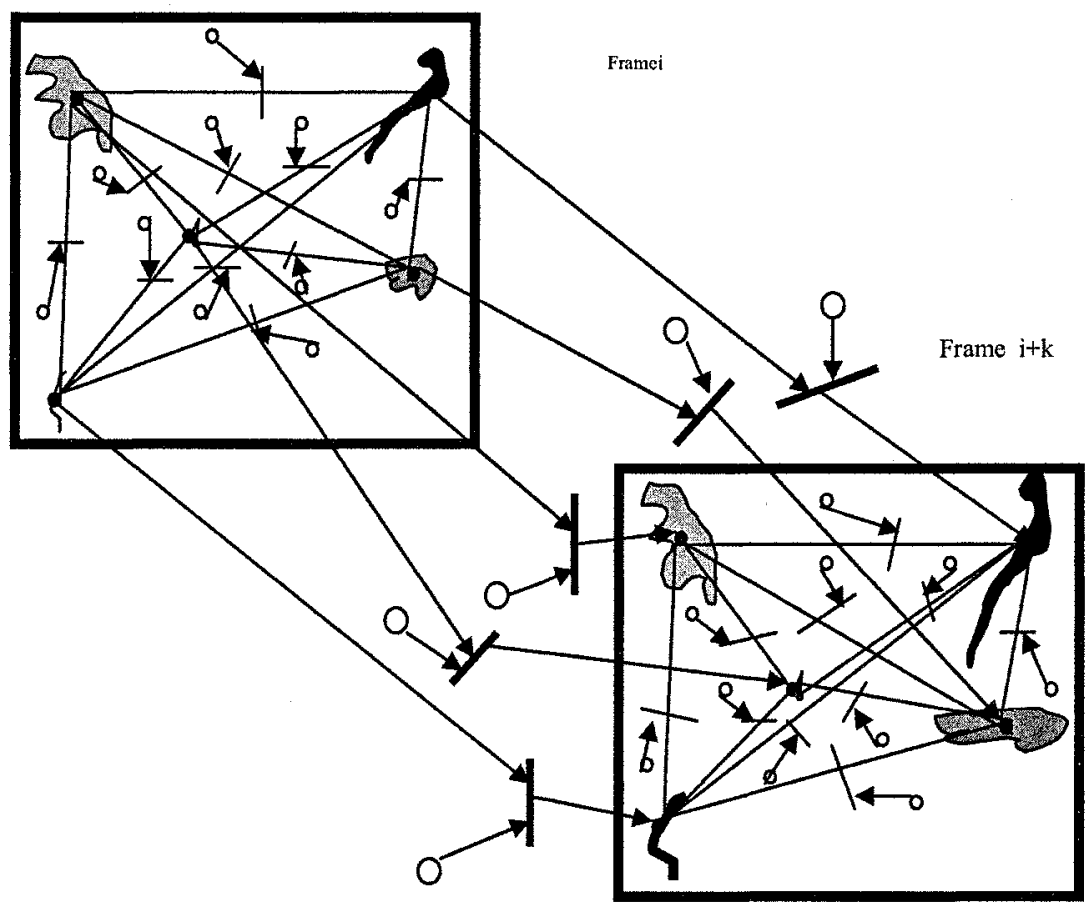




\section{Conclusions}

The extraction of bio-signatures (structural, functional) was the subject described in this paper. The bio-images were captured by a microscope/video camera. This methodology is used for the Bio-signatures project sponsored by AIIS Inc. for recoding and interrelating the behavior (changes) of cancerous cells.

\section{References}

1. M. Kutter and F. A. P. Petitcolas, "A fair benchmark for image watermarking systems", Electronic Imaging '99. Security and Watermarking of Multimedia Contents, ISOE, Vol. 3657, San Jose, CA, Jan 1999.

2. J. A. Saghri, P. S. Cheatham, a. Habibi, "Image Quality Measure Based on Human Visual System Model", Optical Engineering,Vol. 28, No. 7, July 1989

3. D. J. Fleet and D. J. Heeger, "Embedding Invisible Information in Color Images", Proc. of the ICIP, pp. 532-535, Santa Barbara, California, Oct 1997

4. Mark D. Fairchild, Color Appearance Models, Addison Wesley Longman, Inc., 1998

5. N. Bourbakis, Emulating human visual perception for measuring differences in images using an SPN graph approach, IEEE T-SMC, 32, 2, 191-201, 2002

6. A.Moghaddamzadeh and N.Bourbakis, "A Fuzzy Region Growing Approach For Segmentation of Color Images", Pattern Recognition, Vol. 30, No. 6, pp. 867-881, 1997

7. N.Bourbakis, Detecting differences in sequences of images using PFF and LGG, IEEE Conf. TAI-2002, Nov. 2002, VA, pp. 355-362.

8. S.Makrogiannis and N.Bourbakis, Stochastic optimization scheme for automatic registration of aerial images, IEEE TAI-2004, FL, Nov.15-17, 2004, pp. 328-336.

9. N.Bourbakis, J.Gattiker and G.Bebis Representing and interpreting human activity and events from video, Int. JAIT, vol.12,1,2003.

10. N.Bourbakis, Emulating human visual perception for measuring differences in images using an SPN graph approach, IEEE T-SMC, 32, 2, 191-201, 2002. 